\title{
Optimised Tail-based Routing for VANETs using Multi-Objective Particle Swarm Optimisation with Angle Searching
}

\author{
Mustafa Qasim AL-Shammari ${ }^{1}$, Ravie Chandren Muniyandi ${ }^{2}$ \\ Center for Software Technology and Management \\ University Kebangsaan Malaysia, UKM Bangi \\ Selangor - 43600, Malaysia
}

\begin{abstract}
Routing protocols for vehicular ad hoc networks (VANETs) are highly important, as they are essential for operating the concept of intelligent transportation system and several other applications. VANET Routing entails awareness about the nature of the road and various other parameters that affect the performance of the protocol. Optimising the VANET routing guarantees optimal metrics, such as low E2E delay, high packet delivery ratio (PDR) and low overhead. Since its performance is of multi-objective nature, it needs multi-objective optimisation as well. Most researchers have focused on a single objective or weighted average for multi-objective optimisation. Only a few of the studies have tackled the actual multi-objective optimisation of VANET routing. In this article, we propose a novel reactive routing protocol named tail-based routing, based on the concept of location-aided routing (LAR). We first redefined the request zone to reduce the lateral width with respect to the lateral distance between the source and destination and named it tail. Next, we incorporated angle searching with crowding distance inside the multi-objective optimisation MOPSO and called it MO-PSO-angle. Then, we conducted optimisation of tail-based routing using MO-PSO-angle and compared it with optimised LAR, which exhibited the superiority of the latter. The best improvement was at the optimisation point with a $96 \%$ improvement of PDR and a $313 \%$ improvement in E2E delay.
\end{abstract}

Keywords-VANETs; Routing; PDR; E2E delay; optimization; multi-objective particle swarm; location based routing; MOPSO

\section{INTRODUCTION}

Vehicular ad hoc networks VANETs routing is one type of ad hoc networks. It involves connecting vehicles in the road environment for easy communications among them. It has big role in the intelligent transportation system ITS which is considered as an advanced application made to provide innovative services related to modes of transport and traffic management. The result of ITS is accomplishing safety, coordination and intelligence vehicles routing. The relying of ITS on having a reliable and robust VANETs routing is an adequate motivation for researchers to work on solving the issues of VANETs routing. Furthermore, such research can serve in economical saving due to the avoidance of traffic jam and the management of hazardous situations in order to limit the damages or the loss of human life because of road accidents. Routing protocols (RPs) play an essential role in the efficacy of an ad hoc network [1], [2], [3] and [4]. Lack of fixed infrastructure for the ad hoc network makes it a tough task to route a message from a source to its destination. While this process is easy in the case of a traditional network due to the prior availability of the network graph, it is a challenging task in the case of the ad hoc network that has a non-fixed topology. Furthermore, RPs become more challenging in the vehicular ad hoc networks (VANETs) because of the high mobility and associated dynamics of the vehicles. The need is to have a reliable and robust routing protocol that facilitates numerous tasks and applications required to operate a routingdependent application. This becomes more important in the applications of intelligent transportation systems where fast information exchange among vehicles must be enabled. The information exchange is important for providing various traffic and safety services [4]. The literature describes a wide range of routing protocols originated from various views and philosophies. While some approaches are based on the concept of prior preparation of the route or proactive way [5], others are based on the reactive way in which the route is prepared only on demand [6]. In emergency situations or when fast delivery of message is required, proactive routing is preferred. On the other hand, when there is no restriction or constraint on the time of delivery, on-demand or reactive routing protocol is preferred for its low overhead. In either way, the performance is subject to change based on the range of parameters that have to be carefully selected depending on the optimisation approach. Many researchers have adopted or adapted various meta-heuristic optimisation approaches for selecting the best values of parameters. However, a majority of them relied on a single objective optimisation approach which affects the multiobjective nature of the problem [7]. We propose a routing protocol for VANET and optimise it for the multi-objective nature of the problem, offering more control of the performance and flexibility in responding to the need of the user or other applications that are based on the routing.

Optimisation algorithms based on heuristic searching are a big family, including a wide range of approaches such as genetic [7], particle swarm optimization [8], simulated annealing [9], ant bee colony [10], harmony searching [12] and many others [11]. Each of the approaches is inspired by a certain type of world phenomenon or metaphor. Some of them have multi-objective variants such as non-dominated sorting for genetic algorithm, and multi-objective particle swarm optimization [12] [13], sea lion optimization [14], multi- 
objective evolutionary algorithm [15]. Each approach has its own capability of searching that differs according to the used set of criteria of evaluating candidate solutions and their relations in the solutions and objective space. The goal of this study is to propose a novel on-demand routing protocol based on the concept of location-aided routing (LAR) and then to conduct multi-objective particle swarm optimisation for a subset of its internal significant parameter. Next, we prove the importance of using non-dominated-based optimisation for improving the routing protocols from the perspective of networks metrics, namely packet delivery ratio (PDR), E2E delay, and overhead.

This research focuses on developing multi-objective optimized reactive routing protocol of VANETs from various perspectives. We are concerned with PDR, E2E delay and overhead. The optimization changes the time period of updating the neighbour zone and the radius of the coverage zone $\mathrm{R}$ in order to accomplish better performance. To the best of our knowledge, this article provides the first multi-objective reactive routing protocol for VANET.

The remaining of the article is organised as follows. In Section II, we present a previous approach. In Section III, we present the proposed tail-based routing. In Section IV, we provide the results and discussion. Lastly, we provide the conclusion and recommendations.

\section{PREVIOUS APPROACHES}

The literature contains numerous approaches for developing an optimised routing protocol for VANETs. For this purpose, some researchers have adapted the theories of meta-heuristic searching. Some others have used the metaheuristic searching for optimising the clustering approach, which will be used as the routing topology. In [16], the enhanced dragonfly algorithm (EDA) was used to minimise the energy utilisation based on the solution of clustering. For avoiding the local optimal, the algorithm was improved by incorporating Cauchy operator. This work can be criticised easily because of the fact that energy is not the top-most priority of VANET network when compared with the more important aspects related to the quality of the found clusters and the performance of routing. The other objective functions need to be incorporated to meet the multi-objective nature of the problem. In [17], meta-heuristic searching was done for optimisation, and a reputation-based weighted clustering protocol was proposed. For optimisation, a vector of various parameters was used, namely Hello_Interval, Election_Interval, ITJ_Interval PRE_Interval, CH_Timeout_ Interval, CM_Timeout_Interval, Cluster_Size, Weight of Distance, Weight of Velocity, Weight of Reputation and Weight of One-Hop Neighbours OHN. They [18] have used weighted sum, which causes the local minima because of the non-convexity of the optimisation curve. Another example of the application of optimisation of routing in VANET is the multi-casting application for countering the broadcast storm that exists in the emergency or hazardous scenarios in VANETs. In [19], an artificial bee colony (ABC) is used for optimising a fuzzy system used for predicting the highestranked link for routing the RREQ message. The solution was based on the fuzzy membership function and rules. For optimisation, four objective functions were used: PDR, E2E delay, throughput, and the number of control packets. However, they were used in a single objective function based on the weighted sum formula. This causes a fall in the local optimal because of the likelihood of non-convexity of the optimisation surface. Bello (2020) has used genetic optimisation for optimising the routing of VANET employing a set of parameters such as transmit power, frequency, and path loss. The objective function used has maximised the route from the perspective of a new major metric named route metric. Obviously, the optimisation does not consider the multiobjective nature of the problem. In [20], a clustering algorithm centred on moth-flame optimisation (MFO) is proposed. The approach is inspired by the movement of moths with respect to the light source. The author has used it for clustering purpose; however, the study did not clearly present the formulation of the multi-objective functions. In [21], a hybrid fuzzy logic and genetic algorithm was developed with the aim of using the fuzzy logic for weight calculation of the multi-objective functions. The application was for service provided in 5G VANETs. The approach aims at maximising the capacity and the number of fog controller base band unit controllers (FCBBUCs) and at minimising the delay, the number of FC-ZCs that one BBUC handles and the traffic load of each FC-ZC, and consequently of each BBUC pool. It optimises connections between the FC-BBUCs and the FZ-ZCs using the hybrid fuzzy genetic. In [22], a clustering algorithm based on ant colony optimisation (ACO) was proposed. The approach uses two objective functions: delta difference value of the clusters and the summation of the distance values of all CHs from their cluster members. It also uses a weighted sum approach for the two objective functions. This causes local optimality because of the weighted aggregation of the objective functions. In [23], an optimisation for optimised link state routing (OLSR) protocol was proposed. The authors have proposed the use of eight variables for this purpose: HELLO-Interval, REFRESHInterval, TC-Interval, NEIGHB HOLD TIME, HELLOInterval, TOP HOLD TIME, TC-Interval, MID HOLD TIME, TC-Interval, DUP HOLD TIME, WILLINGNESS. The authors have also recommended an objective function that is formulated as weighted equation for the number of packets sent, E2E delay, the number of packets received and throughput. The problem with this approach is the fall in local minima. Some authors have used an optimisation without weighted sum aggregation of the objective functions; they adopted the concept of non-domination. In [24], a nondominated sorting genetic algorithm was used for optimising routing protocol in VANET. OLSR protocol was used for optimisation. However, the authors have used only a small number of parameters in the optimisation, namely hello interval, TC interval, and refresh interval. Furthermore, only two objective functions were used: packet loss and E2E delay. In [25], a hybrid leapfrog algorithm and particle swarm optimisation was proposed for estimating the future position of the nodes and predetermining the link breakage. The specific goal of using PSO is to determine the optimum multiple paths for transmission, while the goal of using the leapfrog mechanism is to obtain the update mechanism. In [26], an optimisation framework for OLSR based on meta-heuristic searching algorithm such as particle swarm optimisation, 
differential evolution, genetic algorithm and simulated annealing was proposed. In order to carry out the optimisation, a formulation of multi-objective function based on weighted summation was done, and a subset of OLSR parameters was selected to optimise the function.

\section{Proposed TAIL BASEd Routing}

This section outlines the developement methodology by first presenting the general block diagram. Further, it provides the neighbour zone update and route request zone. Finally, it describes all the elements of the tail zone, route combining, route reply message, data transmission, route discovery, optimisation, solution space, multi-objective particle swarm optimisation, crowding distance, angle distribution and pseudocode.

\section{A. General Block Diagram}

A block diagram that describes TR, along with an explanation of its elements, is given in Fig. 1. The first block is the neighbour update, which is responsible for building the zone around the node with the purpose of updating the information of nodes around it. Next, the process of the route discovery is performed in which it is combined with three main blocks: tail zone update, sending route request (RREQ) message and receiving route reply message. Then, the data transmission is done based on the selected route. At this stage, the optimisation part changes the parameters of neighbour update and route discovery. For each set of parameters, the network measures are generated and used for evaluating the selected routes. The select parameter block is used to choose one operating route from a set of non-dominated solutions.

\section{B. Neighbor Zone Update}

Each node in the network sends a hello message to its neighbour to update its information. The interval of sending this message is $\mathrm{T}_{\text {hello }}$ and the message is periodic. It includes the following information: the ID of the node, the location information of the node $x$, the velocity of the node $v_{x}$, the acceleration of the node $\mathrm{a}_{\mathrm{x}}$ and hazardous condition. Every hello message includes a timestamp to indicate the moment at which the message was transmitted is given in Table I.

TABLE I. The Topology of the Hello Message

\begin{tabular}{|l|l|l|l|}
\hline ID & $\mathrm{x}$ & $\mathrm{v}_{\mathrm{x}}$ & Timestamp \\
\hline
\end{tabular}

\section{Route Request Message RREQ}

The RREQ message is generated to find a route to a certain destination $\mathrm{D}$. It includes the coordinate information of the source node $\mathrm{x}_{\mathrm{s}}$ and the ID of the node $\mathrm{ID}_{\mathrm{s}}$. It also contains the ID information $I_{D}$, the coordinate information of the node $x_{D}$ and the time stamp at the last update of the RREQ message. The layout of the message is given in Table II.

TABLE II. THE TOPOLOGY OF THE RREQ

\begin{tabular}{|c|c|c|c|c|l|}
\hline $\mathrm{ID}_{\mathrm{s}}$ & $\mathrm{x}_{\mathrm{S}}$ & $\mathrm{ID}_{\mathrm{D}}$ & $\mathrm{x}_{\mathrm{D}}$ & $\mathrm{t}_{\mathrm{D}}$ & Timestamp \\
\hline
\end{tabular}

The RREQ message will be multi-casted to a subset of nodes in the neighbour zone. The subset includes the nodes with high probability to deliver the message. Hence, we select a percentage (LT) that indicates the high probability nodes for delivery. They include the closest LT to the destination based on the expected location of the node. In the case of having a number less than LT in the neighbour zone, the node will send the message to all the nodes in the zone.

\section{Tail Zone}

Let us assume that the nodes are traveling in the highway environment as depicted in Fig. 2. A source node $S$ decides to send data message to a certain destination node named D. Now, an RREQ message will be initiated. The RREQ message will be sent to the neighbour list of nodes $S$. However, for preventing the redundant transmission of messages that could cause flooding, the node will only re-broadcast the message if it exists within the tail zone of the node. The tail zone is defined as the moving rectangular zone that follows the node. As can be seen in Fig. 2, the source node $S$ is supposed to send a packet to the destination node $\mathrm{D}$. The request zone is a tail that starts from the destination node and ends at the proximity of the source node. However, its width is lower than the lateral distance between the source node and the destination. It helps to minimise the broadcast storm of the route request packet and minimise the overhead.

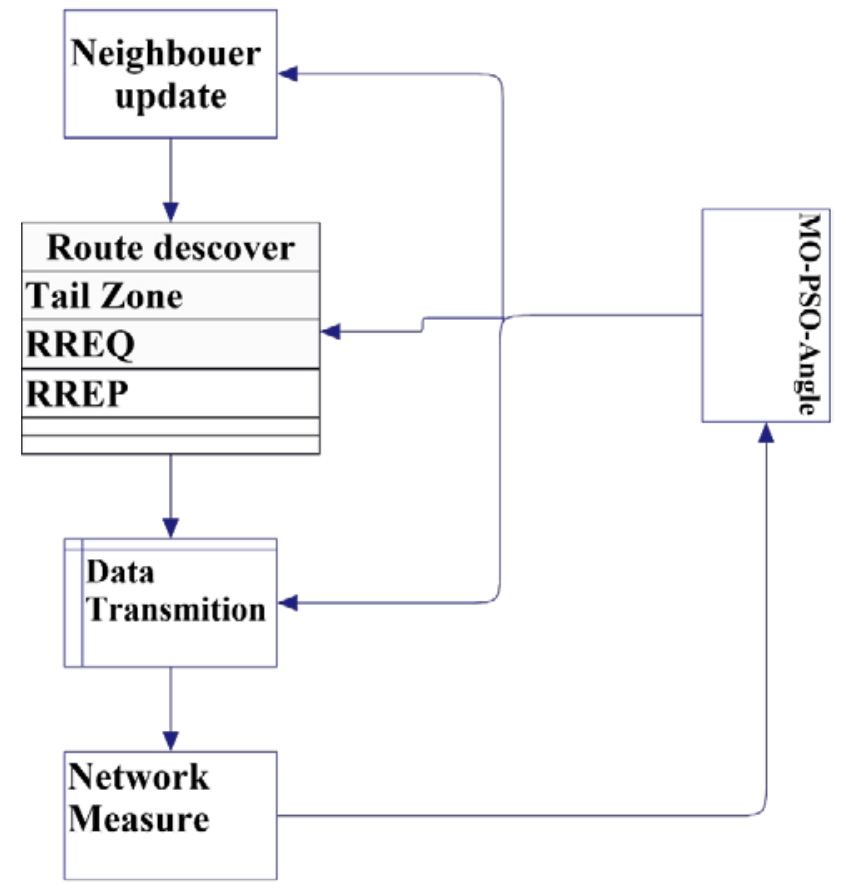

Fig. 1. Block Diagram of Tail-based Routing.

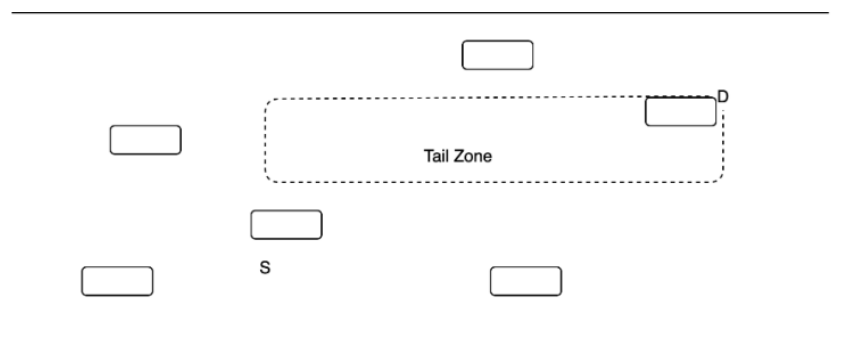

Fig. 2. Examples of Source Vehicle S and Destination Vehicle D and the Tail Zone between them in Tail-based Routing. 
In order for each node to decide whether it is located inside the request zone or outside, it applies the equation.

$x_{D, t}=x_{D, t_{o}}+\left(t-t_{o}\right) v_{D, t_{o}}$

Where

$\mathrm{x}_{\mathrm{D}, \mathrm{t}}$ the location of node $\mathrm{D}$ at $\mathrm{t}$

$\mathrm{x}_{\mathrm{D}, \mathrm{t}_{\mathrm{o}}}$ the location of node $\mathrm{D}$ at $\mathrm{t}_{\mathrm{o}}$

$v_{D, t_{o}}$ the speed of node $D$ at $t_{o}$

This means that any intermediate node $X$ that receives the RREQ message will check the time $t_{o}$ of the last update of the location of $\mathrm{D}$ and compare the time with its own time $t_{0,1}$. It will then update its own information if $t_{0,1}>t_{0}$. Next, the node will use the location information of the source node $\mathrm{S}$ with its own location to make one of two decisions: either to drop the message if the node $X$ is outside the tail zone or to rebroadcast the message if the node $\mathrm{X}$ is inside the tail zone.

\section{E. Route Combining}

At each node, $\mathrm{X}$ receives the RREQ and broadcasts it, and new information is added to RREQ in the form of the ID of the node $\mathrm{X}$ and its current coordinate information. This is for combining a whole path from the destination towards the source for the route reply message.

\section{F. Route Reply Message RREP}

When the RREQ message arrives at its destination, the route reply message is sent from the destination towards the source. This message will go through the reversed route provided in the RREQ. The destination node will consider the first RREQ message that arrives for RREP. Other RREQ messages will be discarded.

\section{G. Data Transmission}

Once a route is established and the route reply message reaches the source, the data transmission will begin from the source towards destination through the path.

\section{H. Route Discovery}

Route discovery is initiated when the node needs to use a route for data transmission. However, the node will use the last used route to the destination if no route error message (REE) is generated for its last use. The REE is generated at the last node that cannot reach its neighbour in the route. In the case of nonvalidity of the route due to REE generated, the node will initiate a new route discovery. The new route initiation will include a new type of RREQ message that contains information towards the last valid node, i.e. the node that generated REE in the last transmission. In this case, the overhead will be reduced because of enabling the valid part in the last discovered route. On the other hand, in the case of non-returning RREP message, the node will not trigger a new route discovery immediately; it will wait for time $\mathrm{T}_{\mathrm{w}}$ or when the condition in the neighbour zone changes to have at least a percentage $\left(P R_{\text {min }}\right)$ of new vehicles entered. The pseudocode that shows the route discovery process is provided in Table III. We assume that the node has a generated packet that needs to be sent to a certain destination. The output is the route that will be used for sending the packet. The process starts with checking the location of the destination node. It is assumed that the location is given in any of the hello messages that the node uses to update its location with respect to its neighbours. Once the location is determined, the node will build the tail zone containing all the nodes that are moving in the road behind the destination node and in the same direction of the destination node. Next, the node will send RREQ message and nodes within the tail zone will have the responsibility of forwarding the packet. The node will wait for the first RREP message which contains the route which will be used to send the packet. If no RREP message is received, the node will wait for a time $\mathrm{T}_{\mathrm{w}}$.

TABLE III. PSEUdocode Of THE Route Discovery PROCESS IN TAIL BASED ROUTING

\begin{tabular}{|ll|}
\hline $1-$ & Input \\
$2-$ & generatedPacket \\
$3-$ & subjectNode \\
$4-$ & Start \\
$5-$ & check the location of the destination node \\
$6-$ & build the tail zone //starting of RouteDiscovery \\
$7-$ & sends RREQ message to the nodes in the tail zone \\
$8-$ & if(receving RREP message) \\
$9-$ & wait for the first RREP message \\
$10-$ & sends the packet in the route of the RREP \\
$11-$ & else \\
$12-$ & wait for Tw \\
$13-$ & end \\
$14-$ & End \\
\hline
\end{tabular}

\section{Optimization}

The goal of optimisation is to improve the performance of the developed protocol to satisfy various aspects of the routing performance measures. Typically, routing needs to satisfy high PDR, less overhead and E2E delay. The implicit conflict of the objectives requires multi-objective optimisation.

\section{J. Solution Space}

For solution space, we consider three variables: the time period of updating the neighbour zone $\mathrm{T}_{\text {hello }}$ and the radius of the coverage zone $\mathrm{R}$.

$\mathrm{x} \in \mathrm{X} \subset \mathrm{R}^{3}$ where $\mathrm{x}=\left(\mathrm{T}_{\text {hello }}, \mathrm{R}, \mathrm{LT}\right)$. The goal is to find the best solution $\mathrm{X}^{*}$ that provides the best performance in terms of the packet delivery ratio, the overhead and the E2E delay. Then, each solution is evaluated by three objective functions

$f_{1}=\operatorname{PDR}(x)$

$f_{2}=-\operatorname{overhead}(x)$

$f_{3}=-E 2 \operatorname{EDelay}(x)$

Adding the constraint of LT $=1$ implies the variant of reduced tail-based routing because the lateral distance of the request zone will be constant. However, we propose another variant where we also optimise $\mathrm{T}_{\text {hello }}, \mathrm{R}$ and the lateral distance of the request zone LT. We call it scaled reduced tail-based routing where $x=\left(T_{\text {hello }}, R, L T\right) x \in X \subset R^{3}$.

We notice that each of the two parameters has an impact on the values of the objective functions. Our goal is to maximise $f_{1}, f_{2}$ and $f_{3}$ for ensuring better performance by increasing the PDR, decreasing the overhead and decreasing the delay. 
Increasing the value of LT implies more overhead; however, it decreases the potential of reaching the destination in a short time, which in turn affects the E2EDelay and the PDR. Hence, there is an implicit confliction between the objectives. This motivates us to use multi-objective optimisation for finding the best solutions.

\section{K. Multi Objective Particle Swarm Optimization MOPSO}

We use multi-objective particle swarm optimisation, which is an extension to the single objective particle swarm optimisation. In this algorithm, a set of initial solutions named as swarm is created and evaluated based on the three objective functions. Next, the non-dominated set of solutions is found and stored in the repository. The algorithm then selects the best global solution and it moves each solution using a mobility equation that combines three effects: inertia, best personal and best global. This is presented in the following equations:

$v_{t}=w v_{t-1}+C_{1} W_{1}\left(x_{b g}-x_{t-1}\right)+C_{2} W_{2}\left(x_{b p}-x_{t-1}\right)$

$x_{t}=x_{t-1}+v_{t}$

$\mathrm{C}_{1}, \mathrm{C}_{2}$ constants

$\mathrm{W}_{1}, \mathrm{~W}_{2}$ random numbers between 0 and 1

\section{Crowding -distance $C D$}

The crowd distance is calculated based on the distance between one of the non-dominated solutions and its two adjacent solutions. Having a higher distance means more potential of exploration. Hence, when we select the solutions, we use the ones with the most crowd distance to choose one iteration over another from the repository that provides the non-dominated solutions.

\section{Angle distribution $A D$}

In addition to the crowd distance, we find the angle between one solution and the other also as a criterion for exploration. We select the solution that has a higher angle between its vector and the two adjacent solutions. This enables more exploration if it is used along with the crowd distance.

\section{N. Pseudocode for Optimization}

For showing how the optimisation works, we present the pseudocode of the optimisation in Table IV. The algorithm receives two inputs: the size of swarm or the number of solutions, NSol and the number of iterations It. The algorithm also receives three coefficients: inertia $\mathrm{w}$, constant of best local $\mathrm{C}_{1}$ and constant of best global $\mathrm{C}_{2}$. Here it is important to state that the best local or global can be set of non-dominated solutions. We need to select one of them as the leader, the maximum velocity $V_{\max }$ and the minimum velocity $V_{\min }$. The output of the pseudocode is the Pareto front which represents set of non-dominated solutions found by the approach. The algorithm starts with the swarm initialization as depicted in line number 11. Next, the algorithm calculates the objective functions of the swarm which is done in the evaluate command in line 12. Afterwards, the algorithm selects the leader as random solution from the non-dominated solution in order to determine the best global which is done in command line number 13. Next, the algorithm initializes the counter of the index of the iteration in line number 15 . Next, the main loop of searching starts in line number 16 and continues until line number 26. This loop represents the main searching loop which is responsible of moving the swarm within the searching space in order to find the non-dominated solutions or the Pareto front that will be reported in line number 29. The loop contains an inner loop that runs on the particles starting from line 17 until line 21. It moves each particle in line 17, mutate the particle in line 18 , evaluate the particle in line 19 , select the best position from the last and the current in line 20, and update the best personal solution of the particle in line 21. Afterwards, the algorithm selects the global leader from the whole swarm in line 23.

TABLE IV. Multi OBJective Particle SWARM Optimization With ANGLE

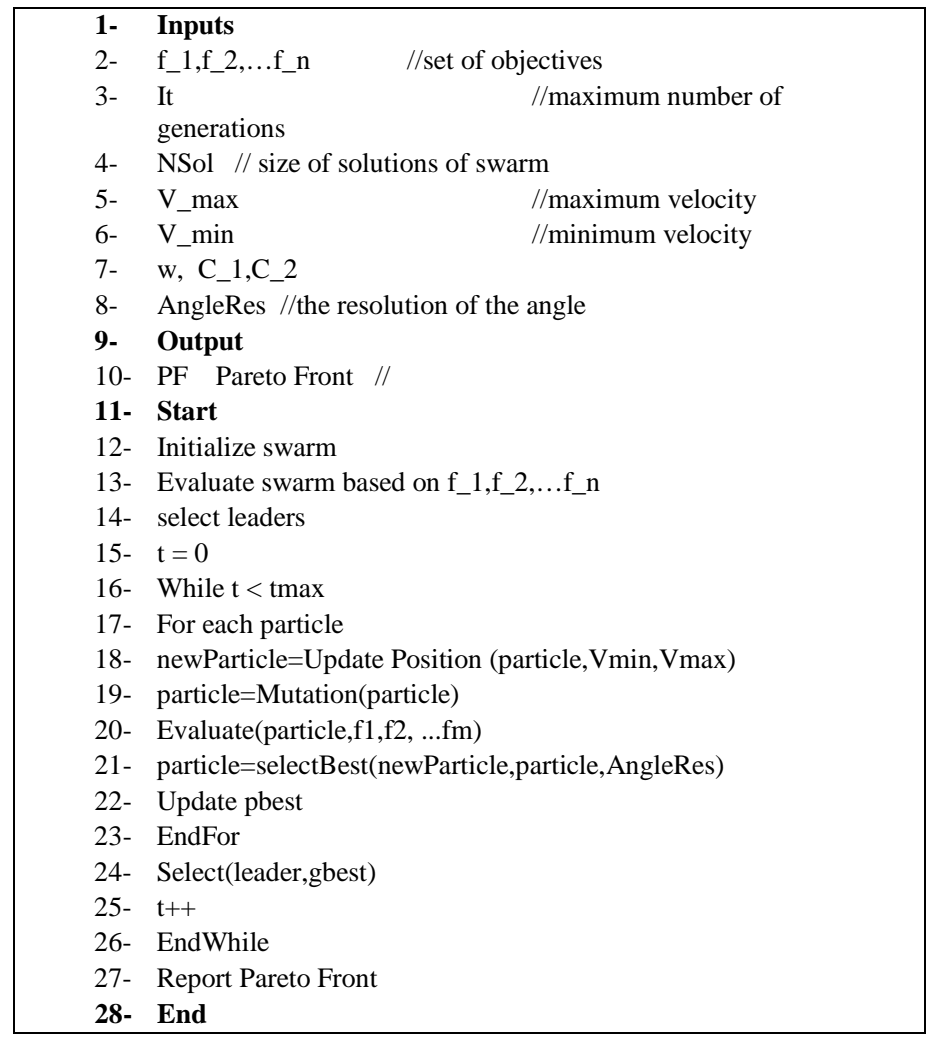

The usage of the angle in the new developed MO-PSOAngle is provided in the calling of procedure of selectBest which put the new particles after moving and the particles before moving in one repository and selects the solutions based on both the crowding distance and the AngleRes in a probabilistic way using pseudocode presented in Table V.

TABLE V. PSEUDOCODE OF SELECTING SOLUTIONS BASED ON THEIR CROWDING DISTANCE AND ANGLE

particle=selectBest(newParticle,particle,AngleRes)

Generate random number $r \in(0,1)$

If $r<0.5$

Selects the solution with the minimum angle density between newParticle and particle

Else

Select the solution with the maximum crowding distance between newParticle and particle

End 
We mean by angle density, the number of solutions inside the angle after decomposing the solution space into angular sectors according to the angle resolution AngleRes. For each solution we define in addition to the crowding distance the angle density which refers to the number of solutions inside the corresponding sector.

\section{RESUlts AND DisCUSSIONS}

For evaluating our developed tail-based routing and MOPSO-Angle, we have implemented both of them and performed an evaluation of simulation of VANETs environment. The optimization was done based on 10 vehicles. The simulation parameters are depicted in Table VI.

TABLE VI. PARAMETERS OF THE SIMULATION

\begin{tabular}{|l|l|}
\hline Parameter name & Parameter value \\
\hline dataPacketLifeTime & $10[\mathrm{sec}]$ \\
\hline interArrivalTime & $6[\mathrm{sec}]$ \\
\hline dataPacketGenerationMean & 2 packets \\
\hline routeRequestTimeOut & $2[\mathrm{sec}]$ \\
\hline routeReplyBufferSize & $100[$ Byte] \\
\hline timeExp & 300 \\
\hline numberOfNodes & $10,20, \ldots 150$ \\
\hline coverageZoneRadius & 100 \\
\hline
\end{tabular}

This section provides the MOO optimization results of LAR, tail based Routing, and Scaled Tail based Routing using MO-PSO-Angle. We have performed 10 experiments for optimizing each of the protocols. We show four of them in Pareto front in Fig. 3. The distribution of the results in the Pareto front show that tail-based routing after optimization was able to provide less E2E delay, less inverse of PDR, and less overhead as the solutions represented by green points are gathered at the corner of the Fig. 3.

However, this is not enough to know quantitatively the performance, then we have to provide the MOO measures.

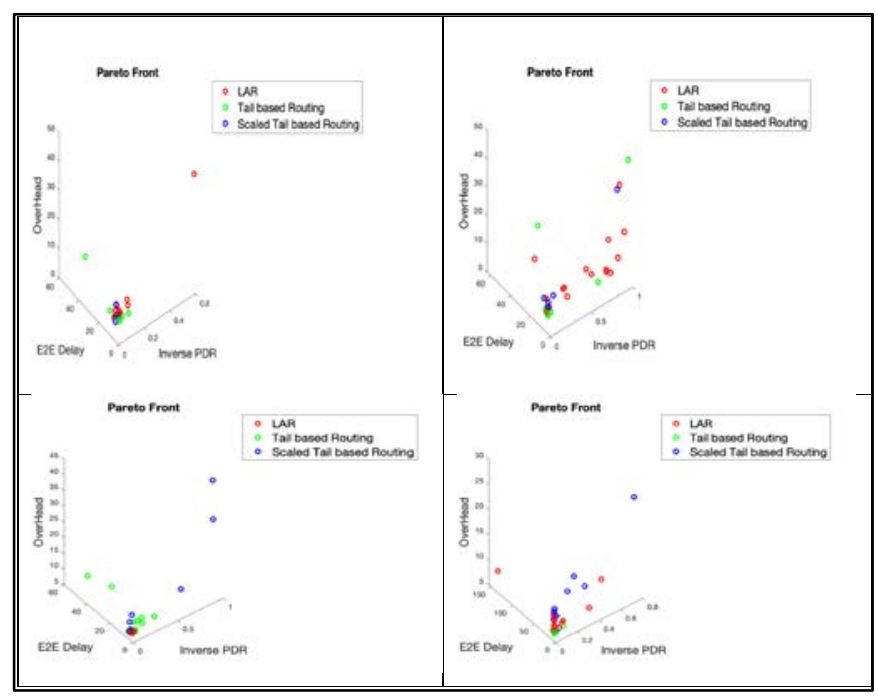

Fig. 3. The Pareto front of LAR, Tail-based Routing and Scaled based Routing.
The visualization of the set coverage in Fig. 4 shows the superiority of tail-based routing over LAR after optimizing, and the superiority of scaled-based routing over LAR after optimizing, however, tail based routing is superior over scaled tail-based routing in terms of obtaining more dominant solutions. This is can interpreted by the fact narrowing the request zone more when we do scaling of it in the scale based tail routing.

In addition to that, we present the results of hyper-volume which shows the spread of the solutions in Fig. 5. We see that tail-based routing has also higher value of hyper-volume. This shows that tail-based routing was able to provide more flexibility of choices to the decision maker for selecting the best point of operation that achieves more domination at the same time in terms of PDR, E2E delay and hyper-volume. In addition, we provide the number of non-dominated solutions for the three protocols after optimization. The results are depicted in Fig. 6.

\section{O. Statistical Evaluation}

The last part that was done is the statistical evaluation which is presented in Table VII. We present 10 experiments ranging from 1 until 9. We provide NDS and HV results for each of the experiments. Next, we apply t-test values between the benchmark LAR, our approach tail-based routing and the scaled tail-based routing which is another variant of our approach. We present the statistical t-test values in Table VIII.

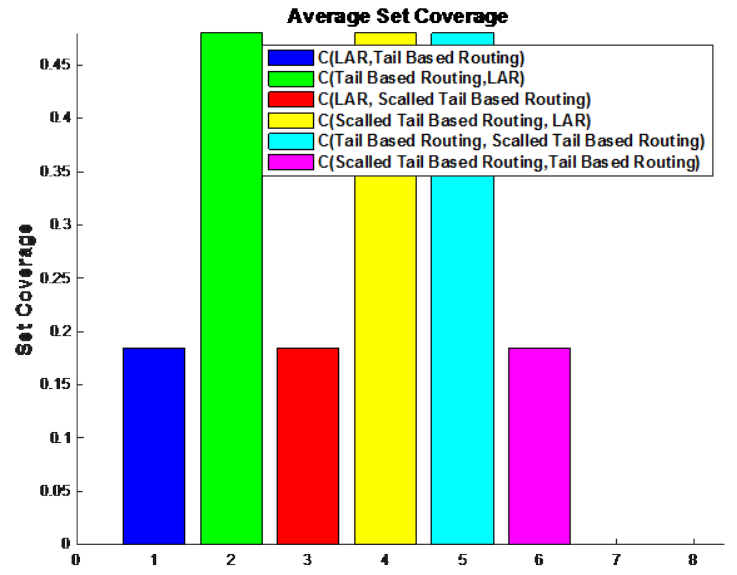

Fig. 4. The Set Coverage of Comparing the Three Protocols: LAR, Reduced Tail based Routing, and Scaled Reduced Tail based Routing.

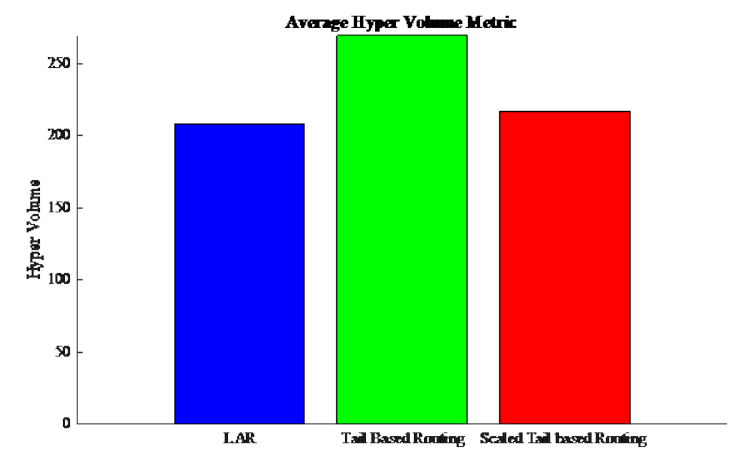

Fig. 5. Hyper-Volume of the Protocols: LAR, tail based Routing and Scaled Tail based Routing. 


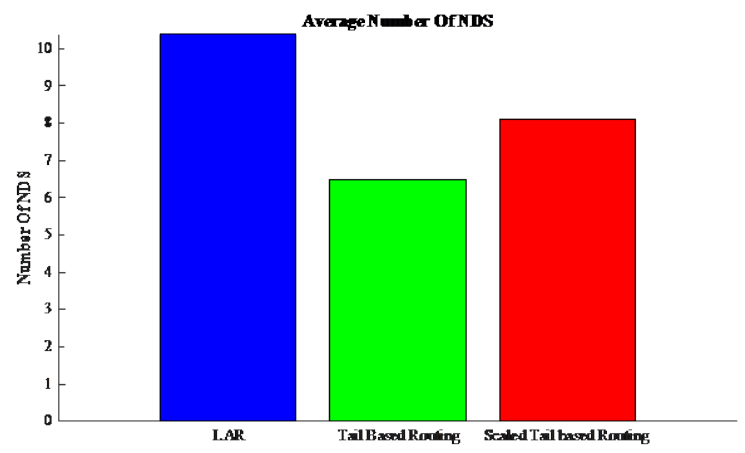

Fig. 6. Number of Non-Dominated Solutions LAR, Tail based Routing and Scaled Tail based Routing.

The results of t-test indicates to superiority in terms of NDS which provides that our developed protocol was competitive from the perspective of NDS considering that is superior in terms of providing more dominating solutions as it is presented in the previous sub-section.

In order to provide the application aspect of the performance of LAR routing and MO-PSO-Angle optimized Reduced Tail based routing, we provide a thorough comparison of the three network evaluation metrics, namely, PDR, E2E delay and overhead for experiments related to the number of nodes which changes from lower value of 30 vehicles up to maximum value of 130 vehicles. Observing Fig. 7, reveals that tail based routing has achieved higher average PDR for all number of nodes that goes from 30 up to 150 . This is interpreted by narrowing down the request zone to small rectangular zone named tail that has more potential of delivering the packets to the destination. This also has implied less E2E delay as it is shown in Fig. 8. We notice that tailbased routing has achieved less E2E delay for the whole possible number of vehicles and in the same range. However, for LAR, E2E delay was high when the number of nodes was low, this shows that LAR is not capable of accommodating for the sparsity in the network due to the smaller number of nodes comparing with tail-based routing. The third performance aspect that has been monitored is the overhead that is shown in Fig. 9 which was only less for a smaller number of nodes while it increased when the number of nodes has increased. This is justified by the fact that the optimization was conducted at low number vehicles (only 10 vehicles) and the system was evaluated based the range from 30 until 150 . This has led to higher value of coverage radius which causes the high overhead comparing with LAR. However, considering that the overhead itself is not a problem when we have low E2E delay and high PDR, we conclude the superiority of tail based routing over LAR. In order to measure the improvement percentage, we use the formula of

$I P=\frac{\text { newMeasure }- \text { OldMeasure }}{\text { oldMeasure }}$

Applying for PDR gives the result of $96 \%$ at 10 nodes and gives for E2E delay the percentage of 313\%.

TABLE VII. STATICALLY EVALUATION

\begin{tabular}{|c|c|c|c|c|c|c|c|c|c|}
\hline Exp No & 1 & 2 & 3 & 4 & 5 & 6 & 7 & 8 & 9 \\
\hline LAR & 228.647522 & 0.16641046 & 0.57624527 & 198.983643 & 58.3471143 & 0.64042105 & 371.524098 & 0.01731366 & $\begin{array}{l}1151.2047 \\
6\end{array}$ \\
\hline Tail based Routing & 272.888518 & 25.451955 & 0 & 2.36052967 & 4.66519149 & 1.14641338 & 0.11795314 & 62.9180172 & $\begin{array}{l}2303.1205 \\
2\end{array}$ \\
\hline $\begin{array}{l}\text { Scaled Tail based } \\
\text { Routing }\end{array}$ & 640.749255 & 72.9114435 & 271.232878 & 41.0532603 & 850.114161 & 0.37399182 & 18.8569746 & 104.647234 & $\begin{array}{l}169.91111 \\
4\end{array}$ \\
\hline LAR & 17 & 4 & 10 & 5 & 11 & 8 & 12 & 4 & 19 \\
\hline Tail based Routing & 8 & 8 & 2 & 4 & 6 & 5 & 5 & 11 & 9 \\
\hline $\begin{array}{l}\text { Scaled Tail based } \\
\text { Routing }\end{array}$ & 16 & 3 & 12 & 12 & 9 & 4 & 9 & 8 & 5 \\
\hline
\end{tabular}

TABLE VIII. T-TEST COMPARISON BETWEEN OUR TAIL BASED ROUTING AND THE LAR AND SCALED FOR HV AND NDS

\begin{tabular}{|l|l|l|l|}
\hline & & LAR & Scaled tail-based routing \\
\hline HV & Tail based routing & 0.61964725 & 0.84418149 \\
\hline & & LAR & Scaled LAR \\
\hline NDS & Tail based routing & 0.10917176 & 0.27551771 \\
\hline
\end{tabular}




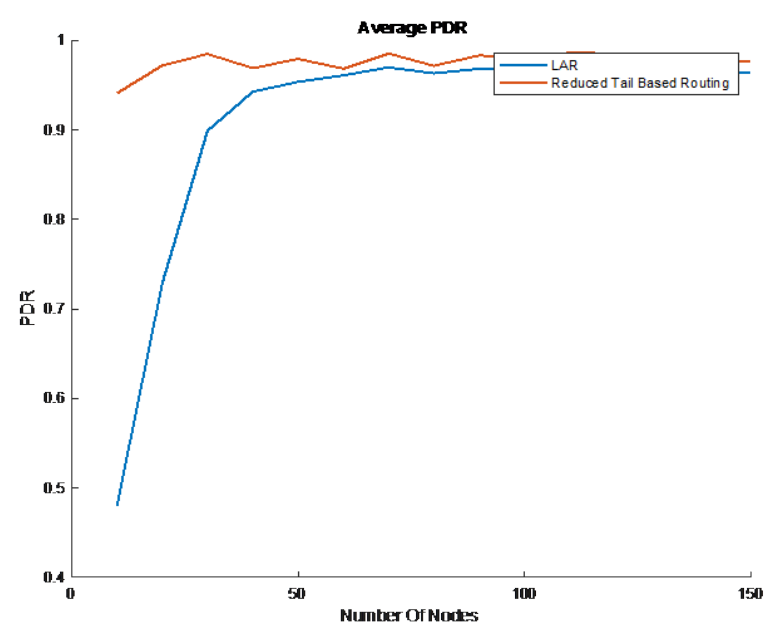

Fig. 7. PDR of LAR and Reduced Tail-based Routing after Optimization using MO-PSO-Angle.

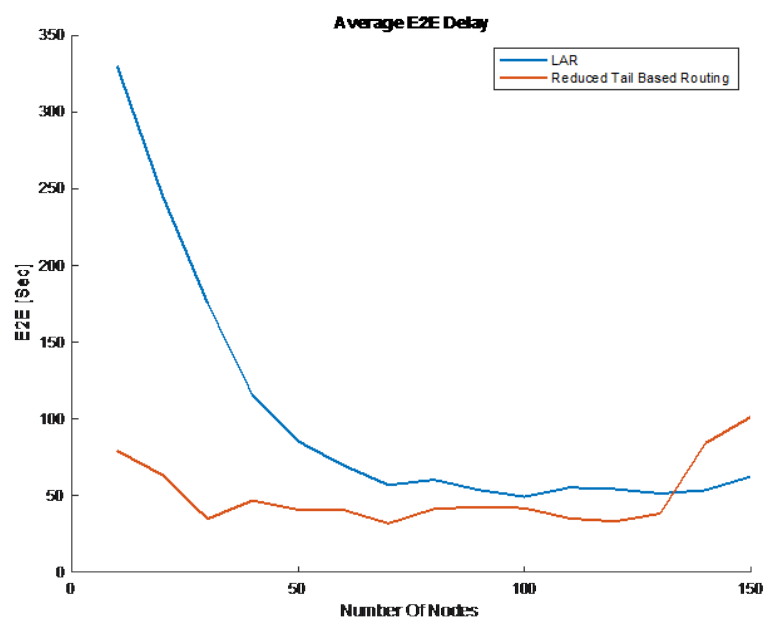

Fig. 8. E2E Delay of LAR and Reduced Tail based Routing after Optimization using MO-PSO-Angle.

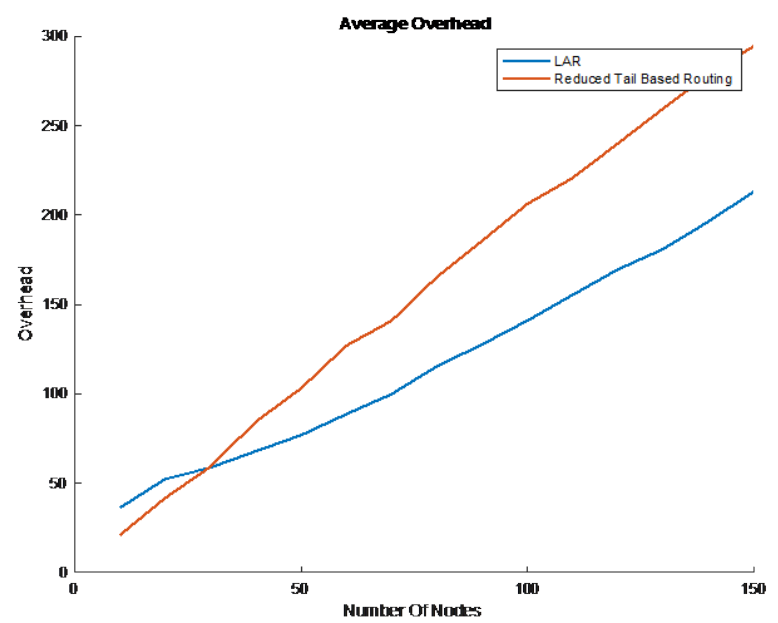

Fig. 9. Overhead of LAR and Reduced Tail-based Routing after Optimization using MO-PSO-Angle.

\section{CONCLUSION AND FUTURE WORK}

This article has tackled the issue of reactive routing protocol in VANETs network. Also, it has provided a new protocol named tail-based routing which is inspired from location aided routing with some modifications. More specifically, it changed the request zone that denotes the region between the source and destination to smaller but more effective region named tail zone which makes it more suitable for highway type of roads. Another aspect that was tackled is the optimization of parameters in the protocols. More specifically, while most researchers conduct single objective optimization with assuming weighted summation is adequate for representing an objective function that combines various needed metrics for optimization in the protocol, we have proposed doing a non-dominated sorting type of optimization. The non-dominated sorting is better to solve the issue of nonconvexity that cannot be resolved in the weighted summation single objective type of optimization. Furthermore, in this thesis we have proposed a novel meta-heuristic searching optimization in the framework of multi-objective particle swarm optimization. This is done by providing a more diversity awareness in the searching by using more than one criterion in the selection of solutions that will be added to the repository. This is done by using angle with crowding distance. We apply it in three protocols: original LAR, our tail based routing and scaled tail-based routing. The results have shown the superiority of tail-based routing when it is optimized using MO-PSO-angle in terms of domination with respect to network measures such as PDR, E2E delay and overhead. Lastly, we have performed statistical comparison with secondary metrics to show the overall competitive performance with respect to them, namely, number of non-dominated solutions and hypervolume. Applying for PDR gives the result of $96 \%$ at 10 nodes and gives for E2E delay the percentage of 313\%.

\section{ACKNOWLEDGMENT}

This research is funded by University Kebangsaan Malaysia (UKM), UKM Grant Code: GGP-2019-023.

\section{REFERENCES}

[1] A. Abbasi, "applied sciences Protocol for VANETs in City Environment," 2018, doi: 10.3390/app8050687.

[2] A. T. A. Naser Abdali and R. C. Muniyandi, "Optimized model for energy aware location aided routing protocol in MANET,” Int. J. Appl. Eng. Res., vol. 12, no. 14, pp. 4631-4637, 2017.

[3] M. A. Jubair et al., "Bat optimized link state routing protocol for energyaware mobile ad-hoc networks," Symmetry (Basel)., vol. 11, no. 11, 2019, doi: 10.3390/sym11111409.

[4] S. Benkerdagh and C. Duvallet, "Cluster-based emergency message dissemination strategy for VANET using V2V communication,” Int. J. Commun. Syst., vol. 32, no. 5, pp. 1-24, 2019, doi: 10.1002/dac.3897.

[5] F. Taha AL-Dhief, R. Chandren Muniyandi, and N. Sabri, "Performance Evaluation of LAR and OLSR Routing Protocols in Forest Fire Detection using Mobile Ad-Hoc Network,” Indian J. Sci. Technol., vol. 9, no. 48, 2016, doi: 10.17485/ijst/2016/v9i48/99556.

[6] G. Zhang, M. Wu, W. Duan, and X. Huang, "Genetic Algorithm Based QoS Perception Routing Protocol for VANETs,” Wirel. Commun. Mob. Comput., vol. 2018, 2018, doi: 10.1155/2018/3897857.

[7] S. Habib, S. Saleem, and K. M. Saqib, "Review on MANET routing protocols and challenges,” Proceeding - 2013 IEEE Student Conf. Res. Dev. SCOReD 2013, no. December, pp. 529-533, 2013, doi: 10.1109/SCOReD.2013.7002647. 
[8] N. Harrag, A. Refoufi, and A. Harrag, "New NSGA-II-based OLSR selforganized routing protocol for mobile ad hoc networks," J. Ambient Intell. Humaniz. Comput., vol. 10, no. 4, pp. 1339-1359, 2019, doi: 10.1007/s12652-018-0947-4.

[9] G. Algorithm, “Genetic Algorithm 4.1,” doi: 10.1007/978-3-319-930251.

[10] D. Wang, D. Tan, and L. Liu, "Particle swarm optimization algorithm : an overview,” Soft Comput., vol. 22, no. 2, pp. 387-408, 2018, doi: 10.1007/s00500-016-2474-6.

[11] L. M. R. Rere, M. I. Fanany, and A. M. Arymurthy, "Simulated Annealing Algorithm for Deep Learning,” Procedia Comput. Sci., vol. 72, pp. 137-144, 2015, doi: 10.1016/j.procs.2015.12.114.

[12] Q. Zhu, X. Tang, Y. Li, and M. O. Yeboah, "An improved differentialbased harmony search algorithm with linear dynamic domain," Knowledge-Based Syst., vol. 187, no. xxxx, p. 104809, 2020, doi: 10.1016/j.knosys.2019.06.017.

[13] H. Shah, R. Ghazali, and N. M. Nawi, "Hybrid ant bee colony algorithm for volcano temperature prediction," Commun. Comput. Inf. Sci., vol. 281 CCIS, pp. 453-465, 2012, doi: 10.1007/978-3-642-28962-0_43.

[14] R. Masadeh, B. A. Mahafzah, and A. Sharieh, "Sea Lion Optimization algorithm,” Int. J. Adv. Comput. Sci. Appl., vol. 10, no. 5, pp. 388-395, 2019, doi: 10.14569/ijacsa.2019.0100548.

[15] Hui Li and Qingfu Zhang, "Multiobjective Optimization Problems With Complicated Pareto Sets, MOEA/D and NSGA-II,” IEEE Trans. Evol. Comput., vol. 13, no. 2, pp. 284-302, 2009, doi: 10.1109/TEVC.2008.925798.

[16] N. Elkhani, R. C. Muniyandi, and G. Zhang, "Multi-objective binary PSO with kernel P system on GPU,” Int. J. Comput. Commun. Control, vol. 13, no. 3, pp. 323-336, 2018, doi: 10.15837/ijccc.2018.3.3282.

[17] C. A. Kerrche, F. Ahmad, M. Elhoseny, A. Adnane, Z. Ahmad, and B. Nour, Emerging Technologies for Connected Internet of Vehicles and Intelligent Transportation System Networks, vol. 242. Springer International Publishing, 2020.
[18] C. J. Joshua, R. Duraisamy, and V. Varadarajan, "A Reputation based Weighted Clustering Protocol in VANET: A Multi-objective Firefly Approach,” Mob. Networks Appl., vol. 24, no. 4, pp. 1199-1209, 2019, doi: 10.1007/s11036-019-01257-z.

[19] T. O. Fahad and A. A. Ali, "Multiobjective Optimized Routing Protocol for VANETs,” Adv. Fuzzy Syst., vol. 2018, 2018, doi: 10.1155/2018/7210253.

[20] S. Yasir, H. Adnan, A. Farhan, K. Fahad, M. Muazzam, and N. Tabassum, "CAMONET: Moth-Flame Optimization ( MFO ) Based Clustering Algorithm for VANETs," IEEE Access, vol. PP, no. c, p. 1, 2018, doi: 10.1109/ACCESS.2018.2868118.

[21] A. A. Khan et al., "A Hybrid-Fuzzy Logic Guided Genetic Algorithm ( H-FLGA ) Approach for Resource Optimization in 5G VANETs,” IEEE Trans. Veh. Technol., vol. 68, no. 7, pp. 6964-6974, 2019, doi: 10.1109/TVT.2019.2915194.

[22] F. Aadil, K. B. Bajwa, S. Khan, and N. M. Chaudary, "CACONET : Ant Colony Optimization ( ACO ) Based Clustering Algorithm for VANET," pp. 1-21, 2016, doi: 10.1371/journal.pone.0154080.

[23] N. M. Al-Kharasani, Z. A. Zulkarnain, S. Subramaniam, and Z. M. Hanapi, "An efficient framework model for optimizing routing performance in vanets,” Sensors (Switzerland), vol. 18, no. 2, 2018, doi: 10.3390/s18020597.

[24] N. Harrag, A. Refoufi, and A. Harrag, "New NSGA-II-based OLSR selforganized routing protocol for mobile ad hoc networks," J. Ambient Intell. Humaniz. Comput., vol. 0, no. 0, p. 0, 2018, doi: 10.1007/s12652018-0947-4.

[25] C. E. Sciences, M. Bhagyavathi, and V. Saritha, "LeapFrog and Particle Swarm Optimization based Multipath Routing for VANETs," vol. 9, no. 31, pp. 1525-1533, 2016.

[26] J. Toutouh, "Intelligent OLSR Routing Protocol Optimization for VANETs,” no. May, 2012, doi: 10.1109/TVT.2012.2188552. 